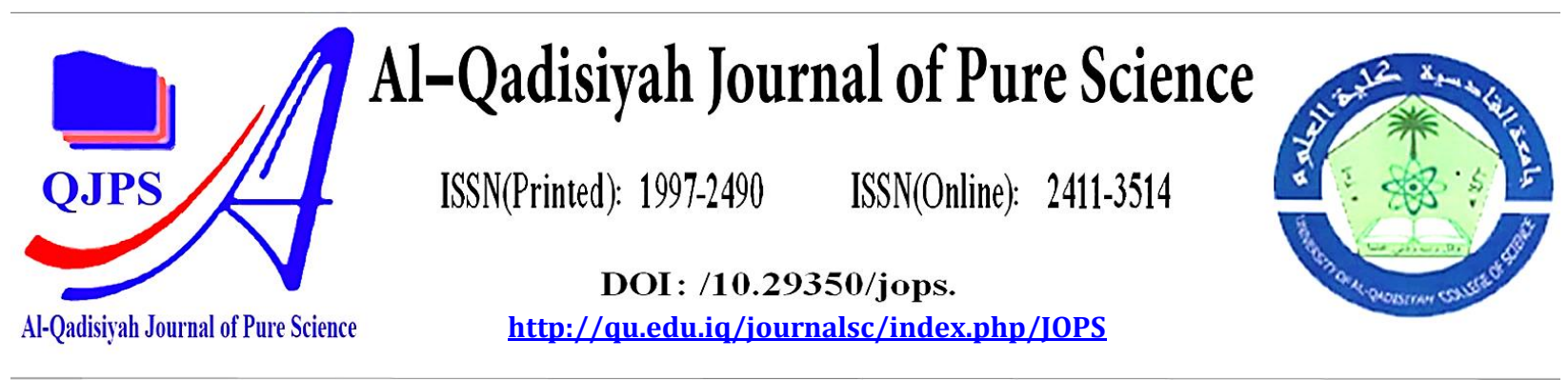

\title{
Synthesis and Characterization ( Oxazepine , Thiazine and Quinazoline ) Derivatives and Study the Biological Activity as Antibacterial
}

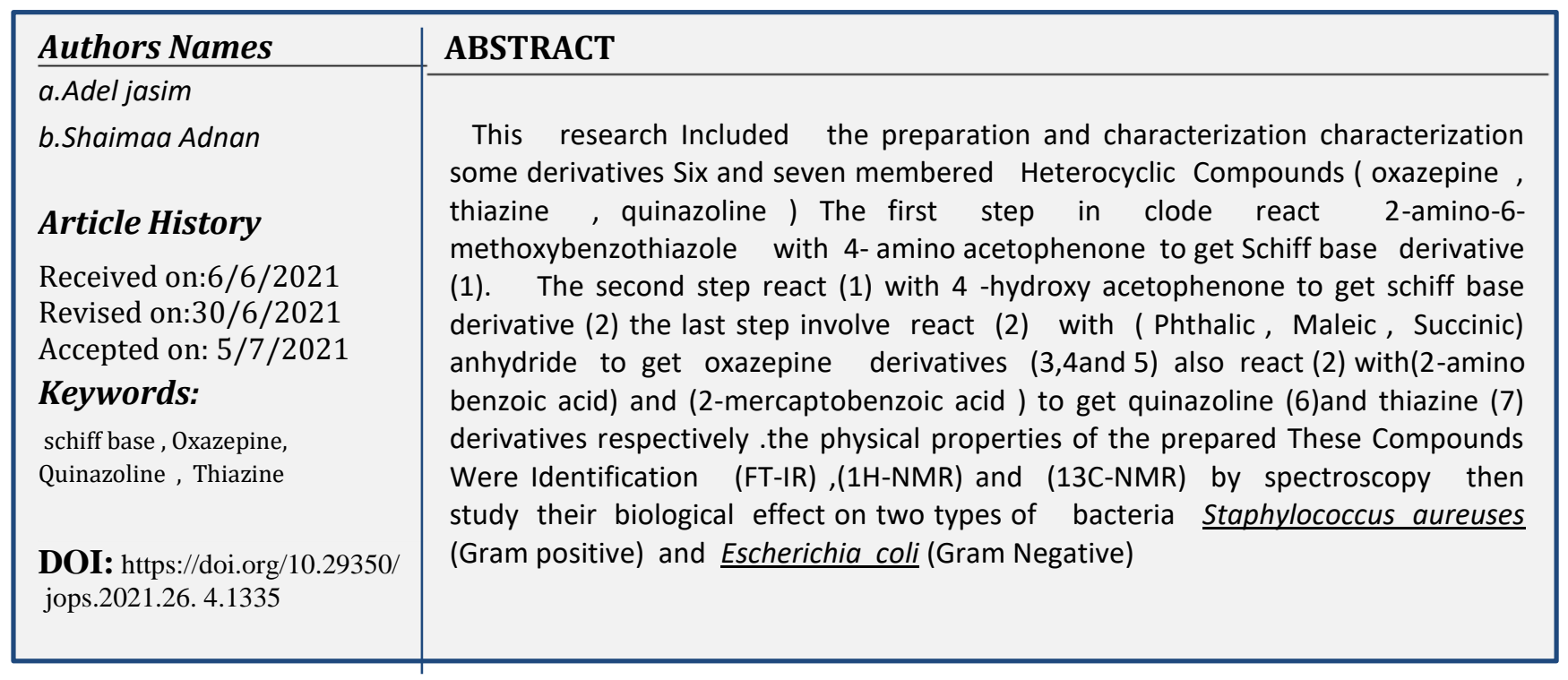

\section{Introduction}

Heterocyclic compounds and their derivatives are considered the most biologically effective because of their great importance in the fields of medicine, as they have been used as antispasmodics, cancerous tumors, bacteria, viruses, and fungi ${ }^{(3)}$. It is part of the life structure of the components of nucleic acids, DNA, RNA, and is also involved in the synthesis of many vitamins and the manufacture of cosmetics and dyes, as well as medicines, dyes and plastics ${ }^{(10)}$. Heterocyclic compounds are also found in hemoglobin (Haem)), which transports oxygen in the blood, as well as the necessary and basic chlorophyll in the photosynthesis ${ }^{(13)}$. Schiff's bases are defined as the products of the chemical reaction of a primary amine with an aldehyde or a ketone under a given set of structural conditions. or azimethine $((\mathrm{C}=\mathrm{N})$ with liberated water molecule which is a feature of schiff bases $^{(7)}$. Is the chief bases is very important as it plays an important role in various fields such as chemistry of inorganic, organic, biological, agricultural and analytical terms are used with some metal ions as pesticides, fungi, manufacture of dyes, corrosion inhibitors and is also characterized by the biological effectiveness of high as anti-cancer ${ }^{(8,9)}$. Many researchers were able to prepare schiff bases, 
including researcher Alam S.A.M. F and his group prepared a variety of (Benz imidazole) derivatives that contain lipid bases and studied their biological effects by Studying their effectiveness on types of bacteria ${ }^{(1)}$. Also, researcher Mieaad and her group (17) were able to prepare a group of SchiffLekand bases and study their biological effectiveness and effect as antibacterials ${ }^{(10)}$. Also, the researcher Slassi and her group were able to prepare several types of azo-shef and study their biological activity as effective anti-fungals ${ }^{(13)}$. Oxazepine derivatives are used as anticancer and ulcerative agents $^{(17,12)}$

\section{Materials}

" (FTIR) Spectra (400 -4000 $\mathrm{cm}^{-1}$ ) in $\mathrm{KBr}$ disk were recorded on SHIMADZU FTIR-8400S Fourier transform. ${ }^{13} \mathrm{C}-\mathrm{NMR}$ and ${ }^{1} \mathrm{HNMR}$ were recorded on Varian Agilent USA at $(500 \mathrm{MHz})$ with (DMSO-d6) measurements were made at Department of Chemistry, Tehran University, Iran."

\subsection{Preparation of the compound $(1)^{(15,5)}$}

Compound No (1) prepares by react $(1 \mathrm{mg}, 0.00739 \mathrm{~m}$ mol) (4-aminoacetophenone) with (1.3333 $\mathrm{mg}, 0.00739 \mathrm{~m} \mathrm{~mol} \mathrm{)} \mathrm{of} \mathrm{(2-amino} \mathrm{-6-} \mathrm{methoxybenzothiazole} \mathrm{)} \mathrm{in} \mathrm{(25} \mathrm{ml)} \mathrm{Ethanol.}$ with, (3 drops) glacial acetic acid(99\%), after which the reflux is done at a temperature of $\left(78^{0} \mathrm{C}\right)$ for a period of (22 hours), and then the solution is left to cool at room temperature for leave. For a period of (24) hours, after then recrystallized .The course of the reaction was monitored By use, technique thin layer chromatography (TLC)

\subsection{Preparation of the compound (2)}

The compound (2) Schiff base was prepared by reacting (1 mg, $0.00336 \mathrm{~m} \mathrm{~mol} \mathrm{)} \mathrm{of}$ compound (1) with $(0.456 \mathrm{mg}, 0.00336 \mathrm{~m} \mathrm{~mol})$ of (4- hydroxy-acetophenone) in (25 ml) of ethanol and then (3 drops) ) of glacial acetic acid, and reflux at a temperature of $\left(78^{0} \mathrm{C}\right)$ for a leave of (26 hours), after which the solution is left to cool at room temperature for a period of (24) hours, and the sediments are recrystallized With methanol

\subsection{Preparation of compound(Oxazepine) (general metode ) $(3,4,5)^{(4)}$}

The three derivatives (Oxazepine) were prepared by reacting $(0.6 \mathrm{mg}, 0.001444 \mathrm{mmol})$ of compound (2) with each of $(0.4277 \mathrm{mg}, 0.001444 \mathrm{~m} \mathrm{~mol})$, (phathalic anhydride), $(0.2831 \mathrm{mg}$, $0.001444 \mathrm{~m} \mathrm{~mol}$ ) (maleic anhydride), ( $0.289 \mathrm{mg}, 0.001444 \mathrm{mmol}$ ) (Succinic anhydride) each dissolves in $(30 \mathrm{ml})$ of dry benzene. The reflux was done from (52-64) hours at a temperature of $\left(80^{0} \mathrm{C}\right)$ after that the solution is left to cool down for a period of (24 hours), After that, it is filtered and recrystallized with ethanol

\subsection{Preparation of compound (6) ${ }^{(16)}$}

Compound (6) is prepared by adding (0.6 mg ) $(0.001444 \mathrm{~m} \mathrm{~mol})$ of compound (2) dissolved in (20) $\mathrm{ml}$ (1.4 dioxane) to $(0.369 \mathrm{mg})(0.001444 \mathrm{~m} \mathrm{~mol})$ of (2-amino benzoic acid) by adding (3 ml) ) Of DMF and then it is reflux (64) hours at a temperature of $\left(101^{\circ} \mathrm{C}\right)$, after and $10 \%$ of sodium bicarbonate solution is added, then it is filtered and then recrystallized with a mixture of (ethanol - water) By (1:2) 
Compound (7) is prepared by adding $(0.6 \mathrm{~m} \mathrm{~g})(0.001444 \mathrm{mmol})$ of compound (2) and dissolved in (22 ml) (benzene) to $0.4452 \mathrm{mg}, 0.001444 \mathrm{~m} \mathrm{~mol}$ ) from 2-mercapto benzoic acid ) with the addition of ( $3 \mathrm{ml}$ ) of (DMF) and (5) drops of (Tri ethyl amine), after which it is escalated for a period of $(55)$ hours at a temperature of $\left(50^{\circ} \mathrm{C}\right)$ added $(10 \%)$ of sodium bicarbonate solution, then filtering and then recrystallizing with (1,4-Dioxan)

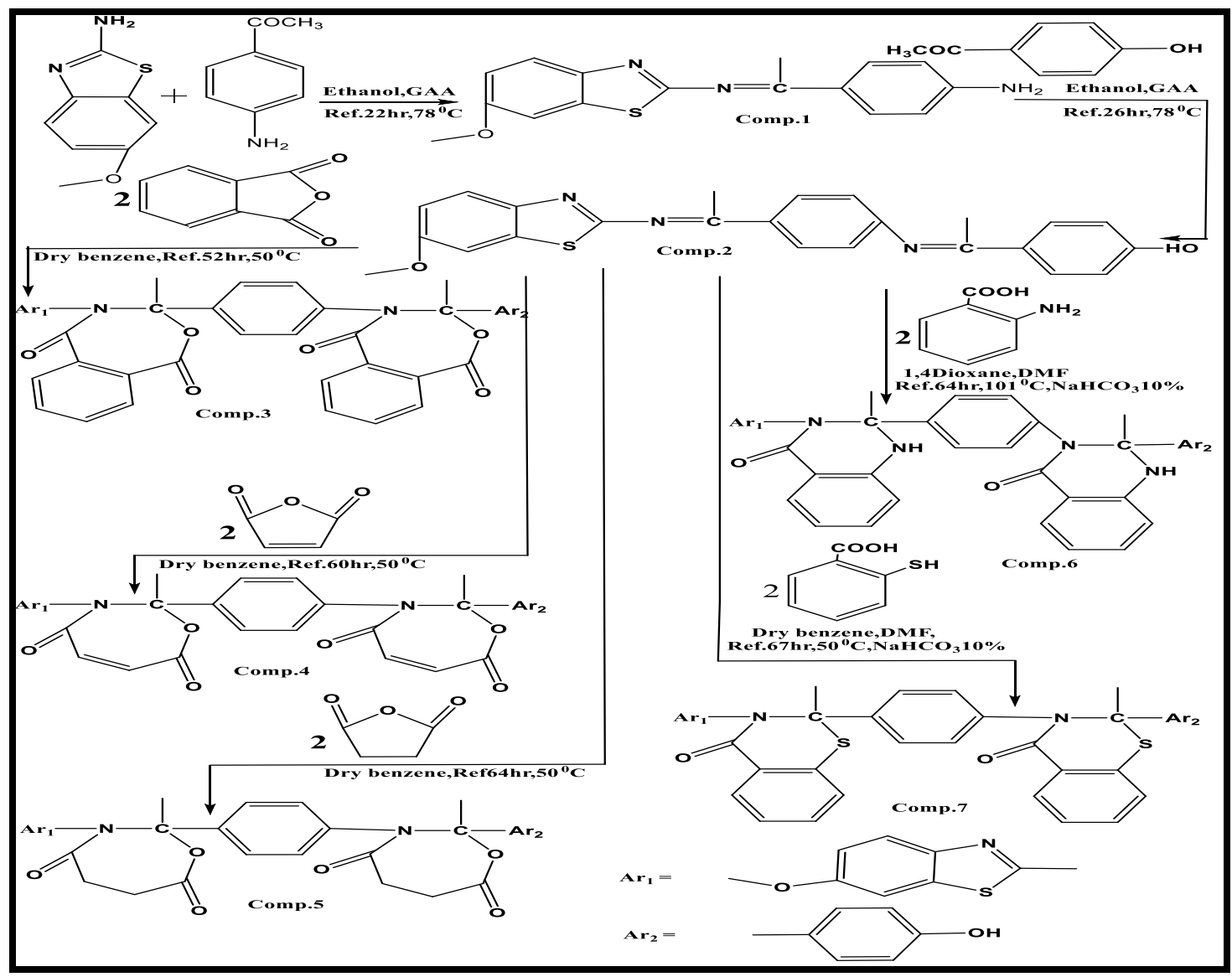

scheme(1) prepare of some heterocyclic derivatives

\section{Results and Discussion}

\section{Characterization of the compound (1)}

\section{4-(1-((6-methoxybenzo[d]thiazol-2-yl)imino)ethyl)aniline}

FT-IR spectrum data for the compound (1) show two peaks at (3491-3353) $\mathrm{cm}^{-1}$ for ($\mathrm{NH} 2$ ) , peak at $3101 \mathrm{~cm}^{-1}$ fo $\mathrm{r}(\mathrm{Ar}-\mathrm{H}), 2947 \mathrm{~cm}^{-1}$ for $(\mathrm{C}-\mathrm{H})$ in $\mathrm{CH} 3,1649 \mathrm{~cm}^{-1}$ for $(\mathrm{C}=\mathrm{N}), 1621$ $\mathrm{cm}^{-1}$ for $(\mathrm{C}=\mathrm{C}), 1272 \mathrm{~cm}^{-1}$ for (C-S). ${ }^{1} \mathrm{HMNR}$ spectrum data of compound (1) show $2.50 \mathrm{ppm}$ (DMSO) , 4.5ppm (S ,2H, (NH2)) , 3. 7ppm (S ,3H, (OCH3)) , $3.3 \mathrm{ppm}(\mathrm{S}, 3 \mathrm{H}, \quad(\mathrm{CH} 3)(6.03-7.69$ ppm (m ,7H, (Ar-H)), . The ${ }^{13}$ CNMR spectrum data (DMSO) compound (1) show : 58 ppm $\left(\mathrm{C}_{15}\right.$ ),167 ppm $\left(\mathrm{C}_{5}\right), 156 \mathrm{ppm}\left(\mathrm{C}_{8}\right), 28 \mathrm{ppm}\left(\mathrm{C}_{16}\right)$ 108-149ppm ( $\left.\mathrm{C}_{\text {Arom }}\right)$ 


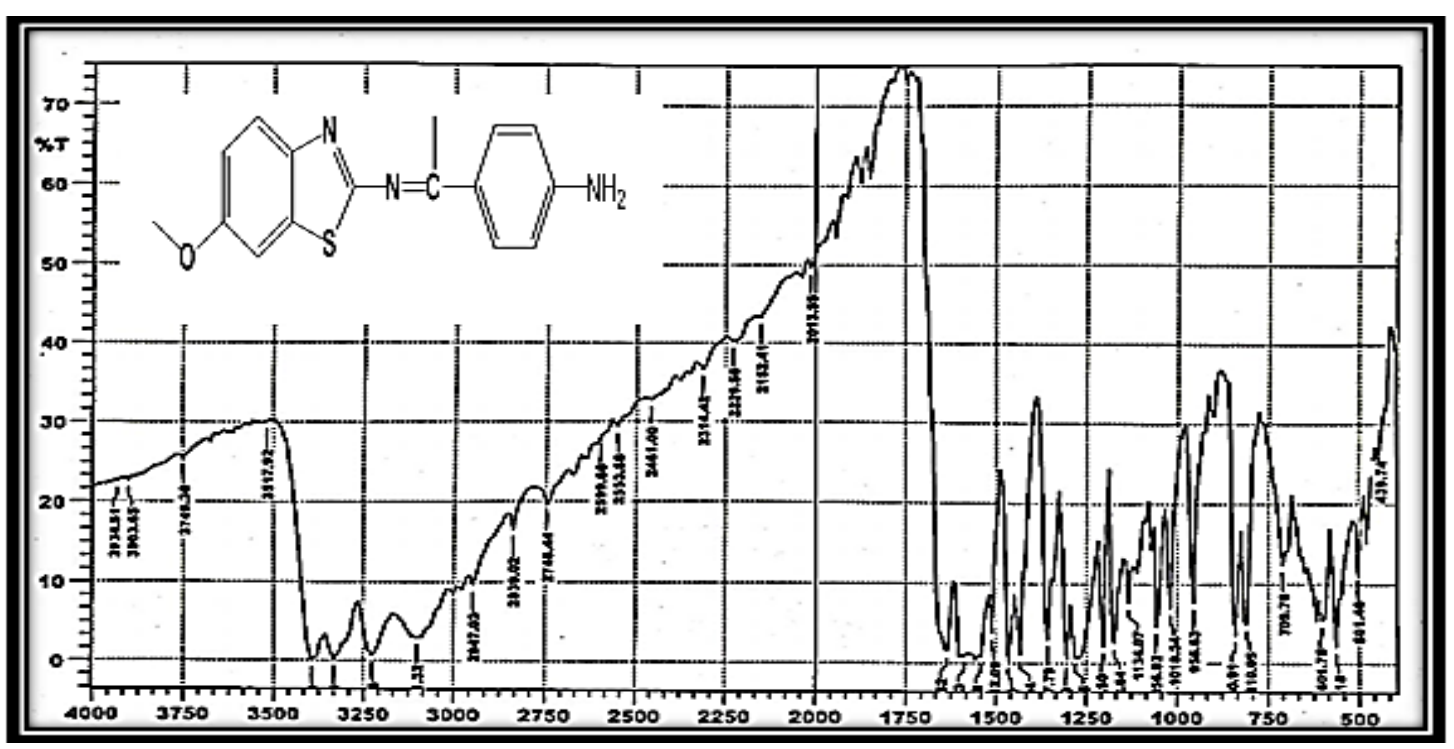

Fig(1) FTIR spectrum of compound(1)
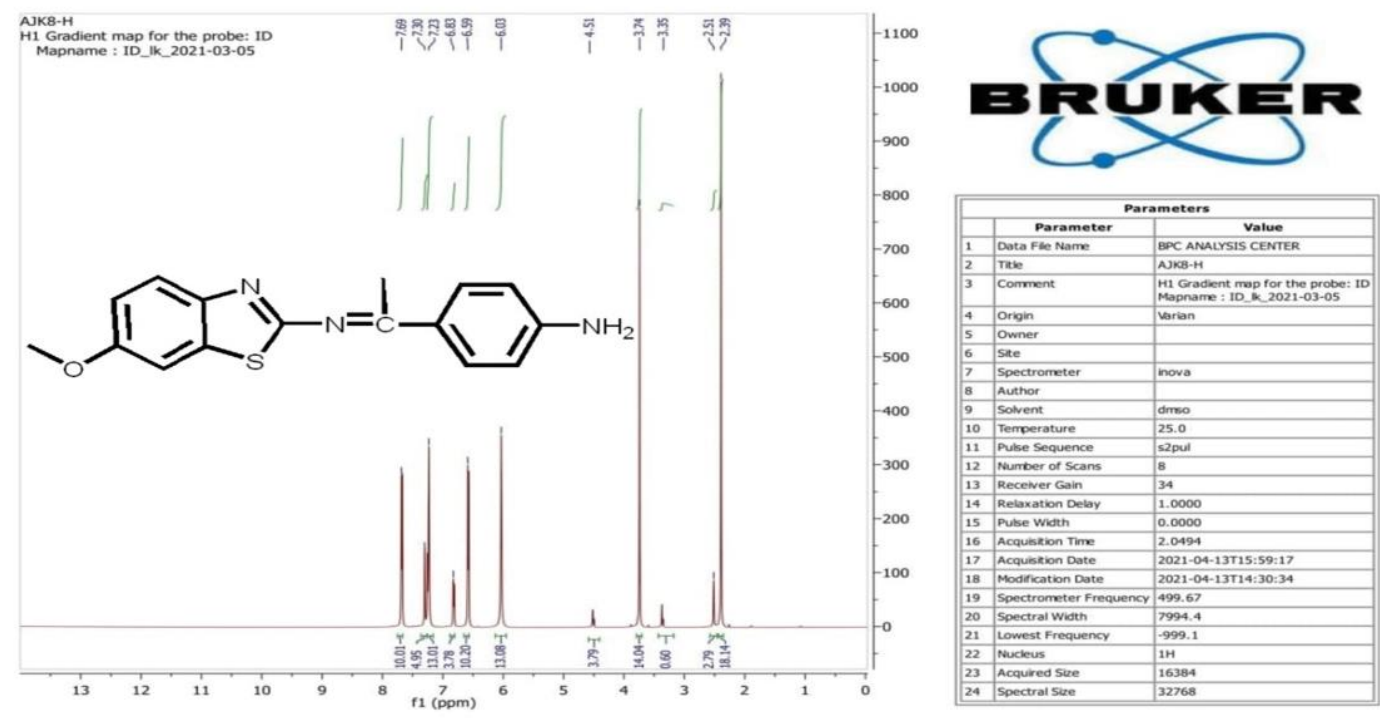

Fig(2)( ${ }^{1}$ H-NMR) spectrum of compound(1) 


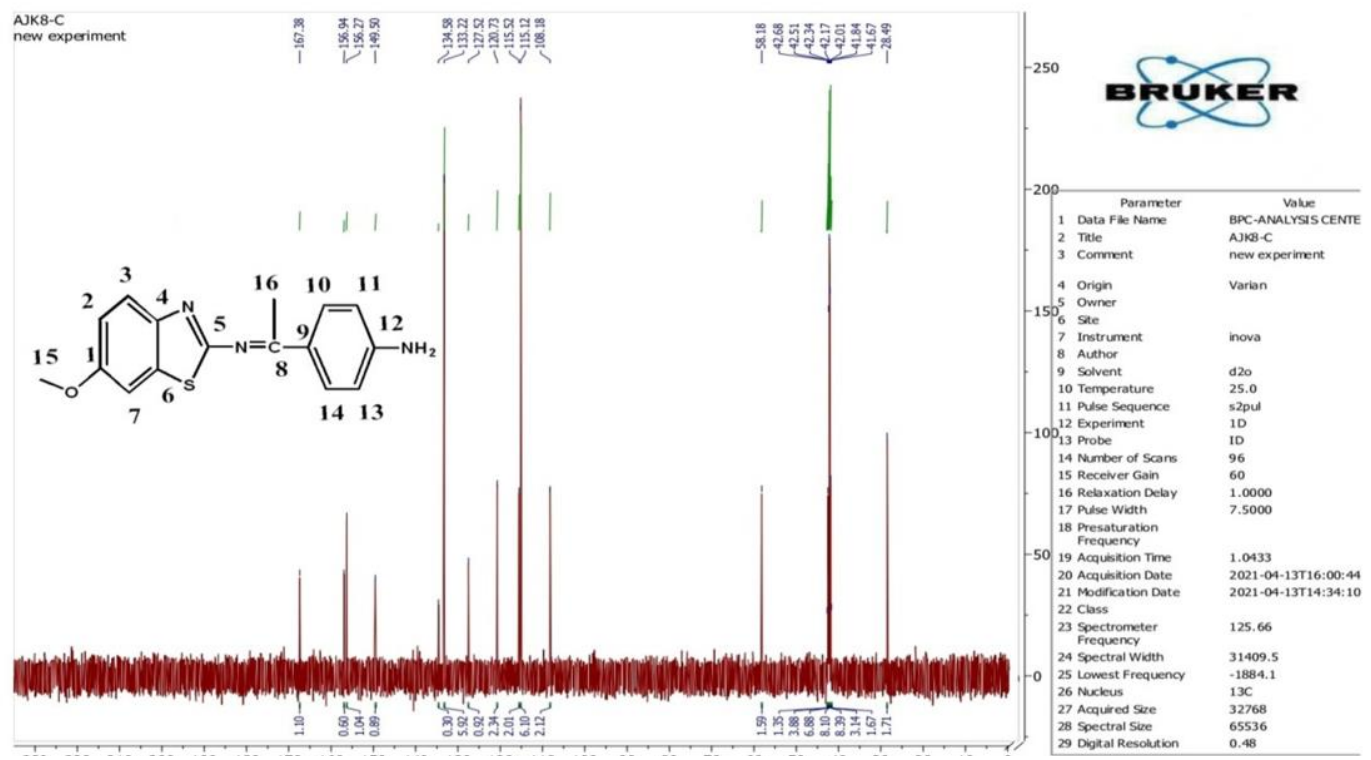

Fig(3) $\left({ }^{13} \mathrm{C}-\mathrm{NMR}\right)$ spectrum of compound(1)

\section{Characterization of the compound (2)}

\section{4-(1-((4-(1-((6-methoxybenzo[d]thiazol-2-yl)imino)ethyl)phenyl)imino)ethyl)phenol}

FT-IR spectrum data for the compound (2) at $3433 \mathrm{~cm}^{-1}$ for $(\mathrm{O} \mathrm{H}), 3101 \mathrm{~cm}^{-1}$ for $(\mathrm{Ar}-$ $\mathrm{H}), 2947 \mathrm{~cm}^{-1}$ for $(\mathrm{C}-\mathrm{H})$ in $\mathrm{CH} 3,1635 \mathrm{~cm}^{-1}$ for $(\mathrm{C}=\mathrm{N}), 1602 \mathrm{~cm}^{-1}$ for $(\mathrm{C}=\mathrm{C}), 1272 \mathrm{~cm}-1$ for $(\mathrm{C}-\mathrm{S})$. ${ }^{1} \mathrm{HMNR}$ spectrum data of compound (2) show $2.50 \mathrm{ppm}$ (DMSO), $10.4 \mathrm{ppm}(\mathrm{S}, 1 \mathrm{H},(\mathrm{OH}))$, $3.7 \mathrm{ppm}(\mathrm{S}, 3 \mathrm{H},(\mathrm{OCH} 3)), 1.9 \mathrm{ppm}(\mathrm{S}, 6 \mathrm{H},(\mathrm{CH} 3)), 6.0-7.8 \mathrm{ppm}(\mathrm{M}, 18 \mathrm{H},(\mathrm{Ar}-\mathrm{H}))$ ). The ${ }^{13} \mathrm{C}-\mathrm{NMR}$ spectrum data ( DMSO) compound (2) show : 58 ppm $\left(\mathrm{C}_{22}\right), 28 \mathrm{ppm}\left(\mathrm{C}_{23}, \mathrm{C}_{24}\right), 164 \mathrm{ppm}\left(\mathrm{C}_{1}\right)$, 139ppm $\left(\mathrm{C}_{15}, \mathrm{C}_{8}\right),, 108-140 \mathrm{ppm}\left(\mathrm{C}_{\text {Arom }}\right)$

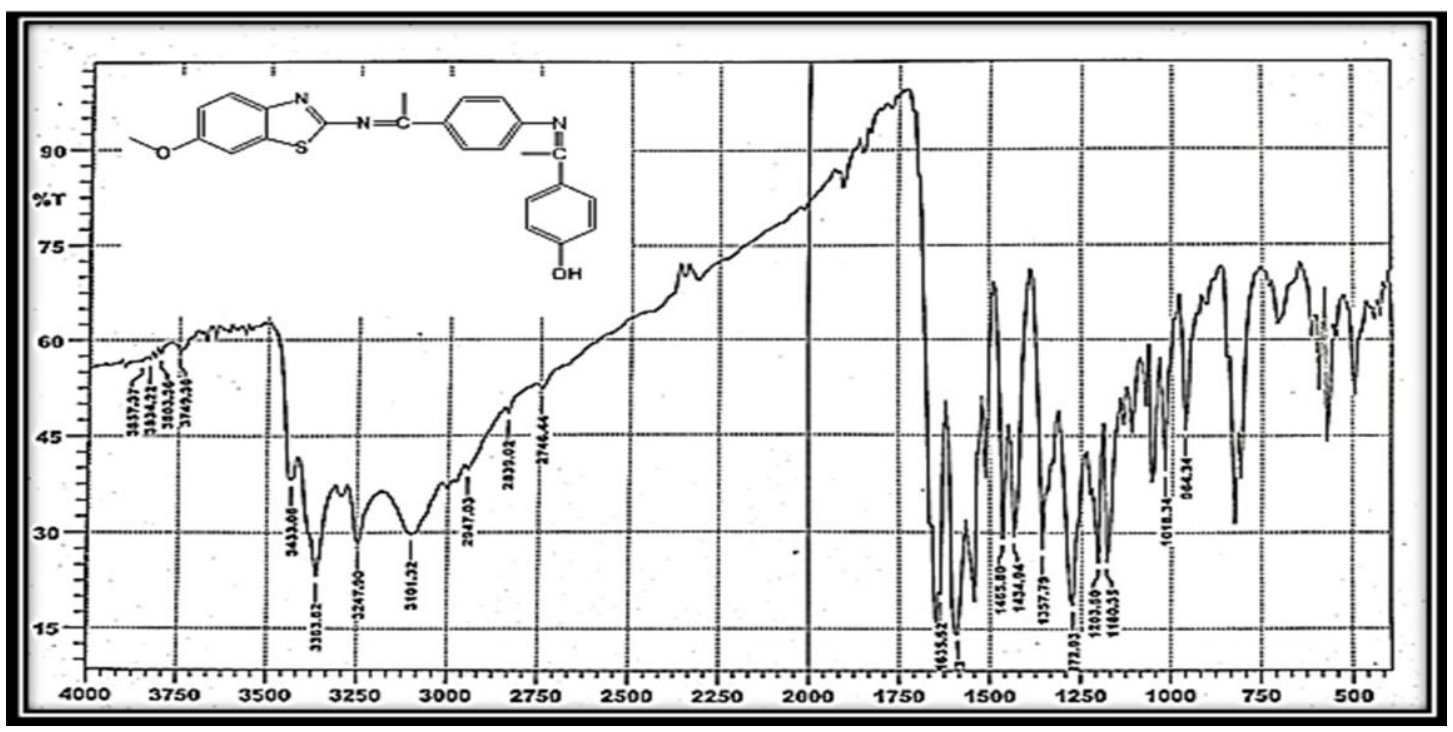

Fig(4) FTIR spectrum of compound(2) 


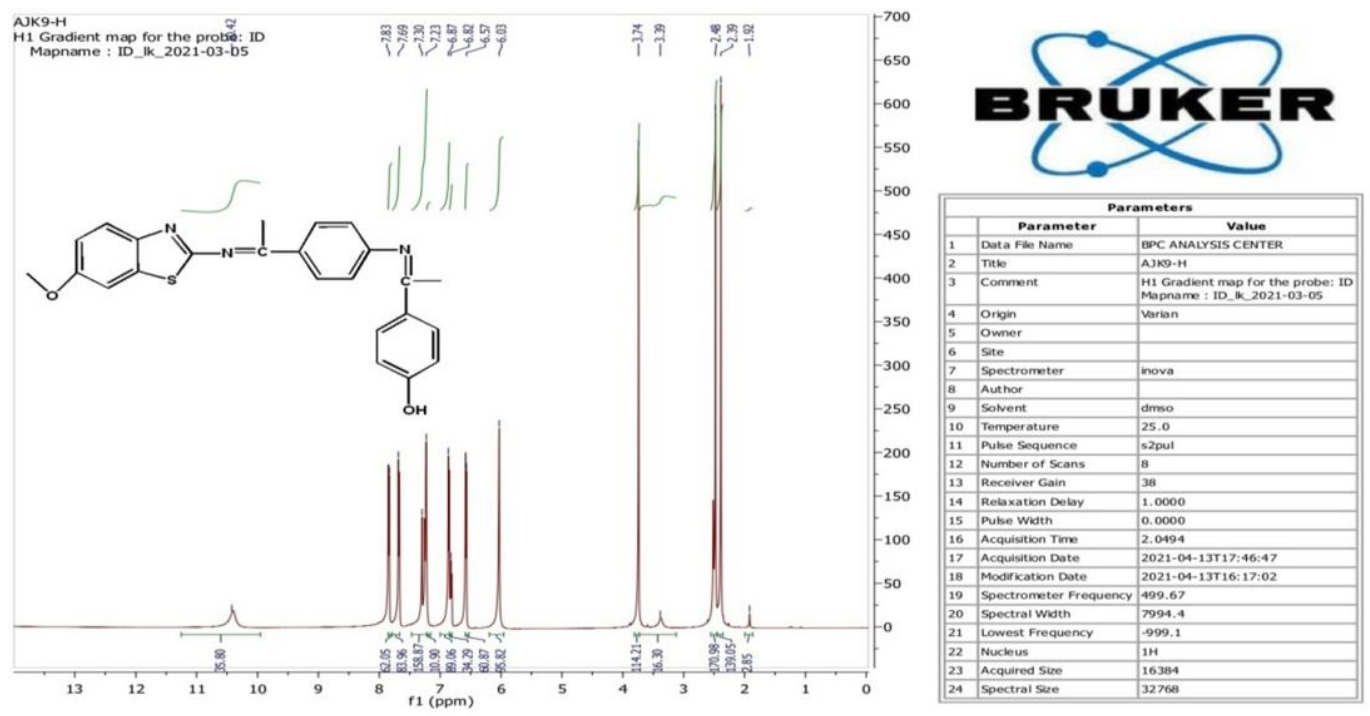

Fig(5)( ${ }^{1}$ H-NMR) spectrum of compound(2)

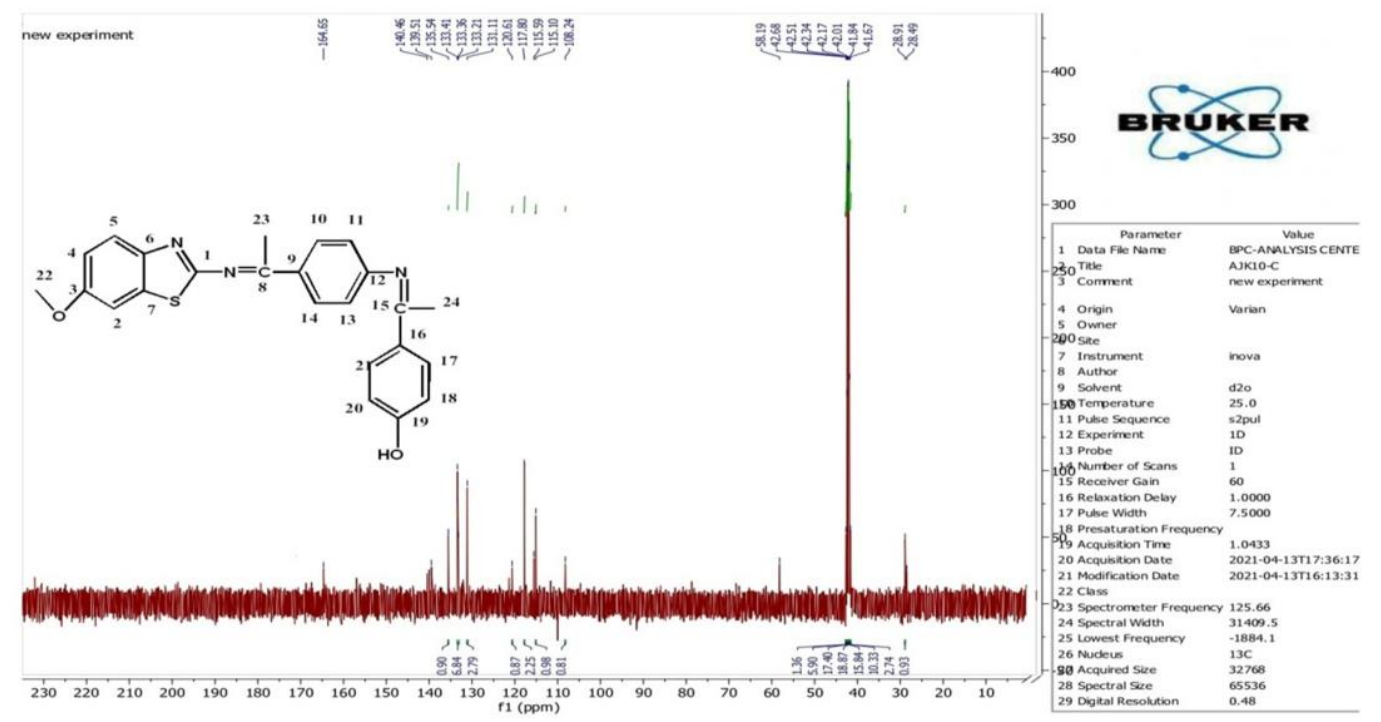

Fig(6) $\left({ }^{13} \mathrm{C}-\mathrm{NMR}\right)$ spectrum of compound(2)

\section{Characterization of compound (3)}

3-(4-hydroxyphenyl)-4-(4-(4-(6-methoxybenzo[d]thiazol-2-yl)-3-methyl-1,5-dioxo-1,3,4,5tetrahydrobenzo[e][1,3]oxazepin-3-yl)phenyl)-3-methyl-3,4-dihydrobenzo[e][1,3]oxazepine-1,5dione

FT-IR spectrum data for the compound (3) show peak at $3355 \mathrm{~cm}^{-1}$ for (OH) $3232 \mathrm{~cm}^{-1}$ for $(\mathrm{Ar}-\mathrm{H}), 2931 \mathrm{~cm}^{-1}$ for $(\mathrm{C}-\mathrm{H})$ in $\mathrm{CH} 3,1666 \mathrm{~cm}^{-1}$ for $(\mathrm{C}=0), 1635 \mathrm{~cm}^{-1}$ for $(\mathrm{C}=\mathrm{N}), 1573 \mathrm{~cm}^{-1}$ for $(\mathrm{C}=\mathrm{C}), 1265 \mathrm{~cm}^{-1}$ for $\quad(\mathrm{C}-\mathrm{S}) .{ }^{1} \mathrm{HMNR}$ spectrum data of compound (3) show 2.50ppm 
(DMSO) $10.3(\mathrm{~S}, 1 \mathrm{H},(\mathrm{OH})), 3.7 \mathrm{ppm}(\mathrm{S}, 3 \mathrm{H},(\mathrm{OCH} 3)), \quad 2.09 \mathrm{ppm}(\mathrm{S}, 6 \mathrm{H},(\mathrm{CH} 3)), 6.5-7.9 \mathrm{ppm}(\mathrm{M}$ ,11H, (Ar-H)),. The ${ }^{13}$ CNMR spectrum data (DMSO) compound (3) show : $197 \mathrm{ppm}\left(\mathrm{C}_{16}, \mathrm{C}_{31}\right)$ ,198 ppm $\left(\mathrm{C}_{9}, \mathrm{C}_{24}\right), \quad 28.4 \mathrm{ppm}\left(\mathrm{C}_{40}, \mathrm{C}_{39}\right), \quad 167 \mathrm{ppm}(\mathrm{C} 1), \quad 58 \mathrm{ppm}\left(\mathrm{C}_{8}, \mathrm{C}_{23}\right), \quad 164 \mathrm{ppm}\left(\mathrm{C}_{35}\right)$ $., 28.8 \mathrm{ppm}\left(\mathrm{C}_{38}\right),(108-156)\left(\mathrm{C}_{\text {Arom }}\right)$

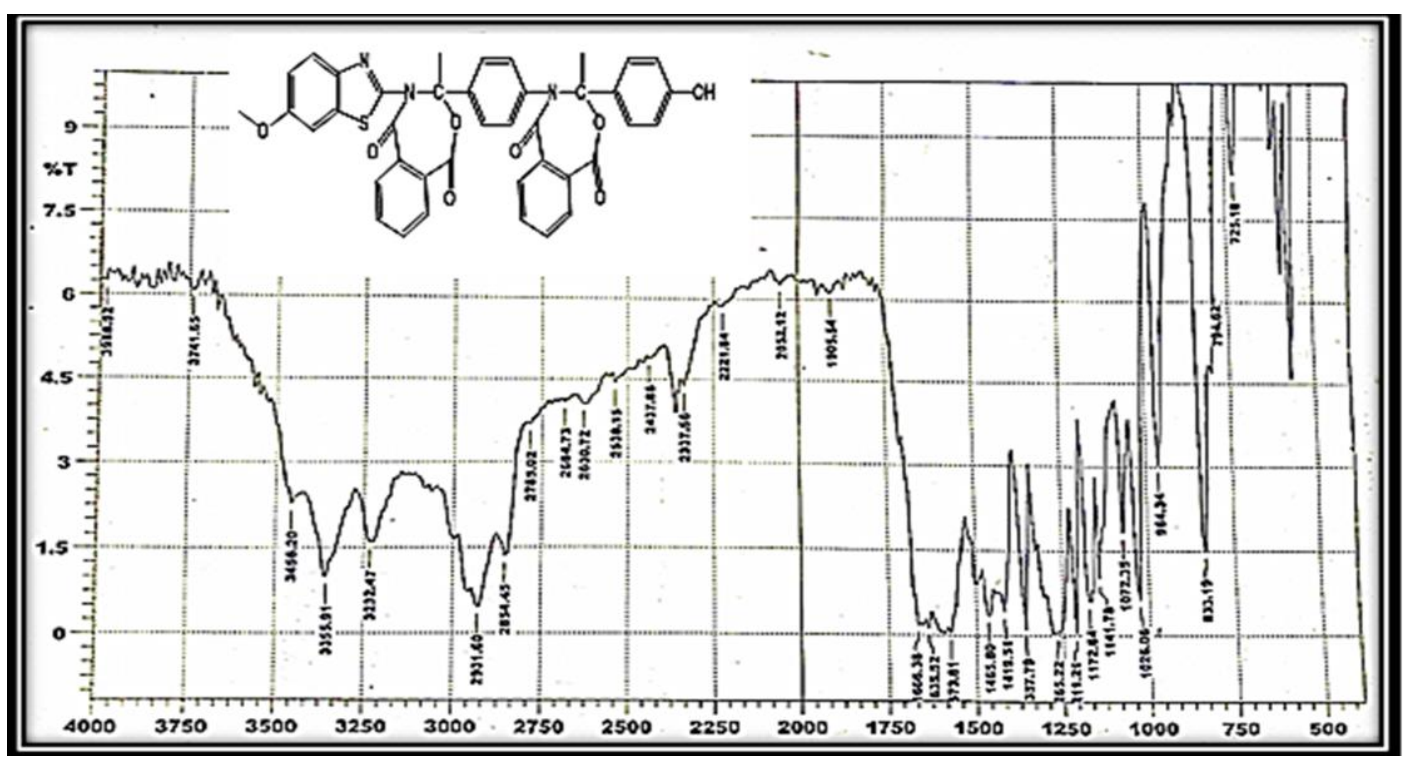

Fig(7) FTIR spectrum of compound(3)

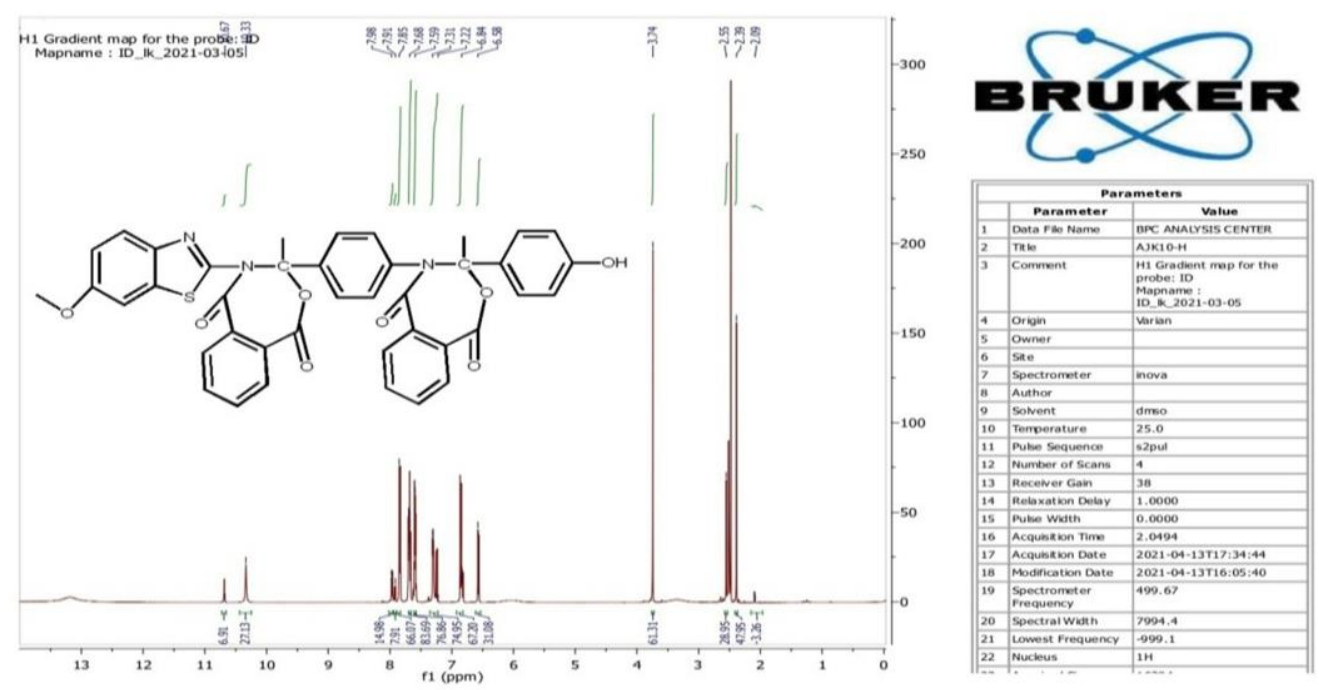

Fig(8)( ${ }^{1}$ H-NMR) spectrum of compound(3) 


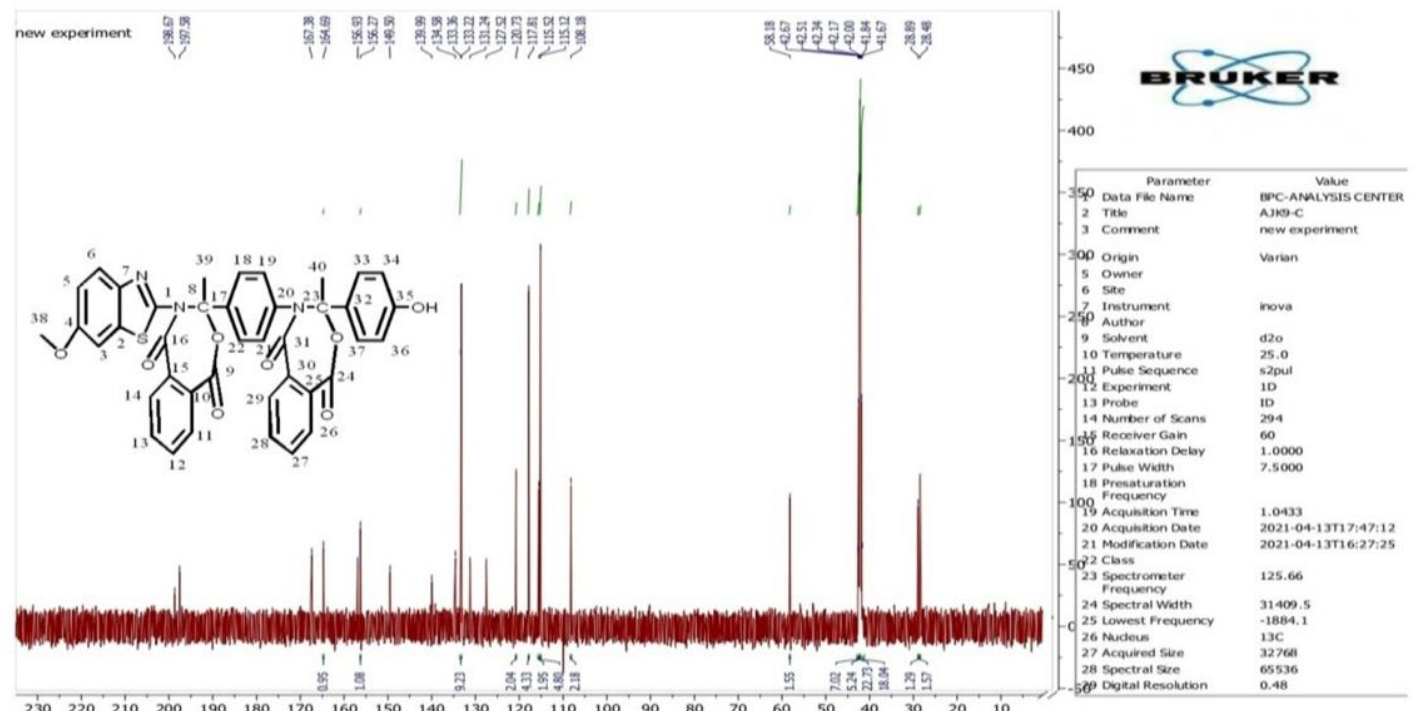

Fig(9) $\left({ }^{13}\right.$ C-NMR) spectrum of compound(3)

\section{Characterization of compound (4)}

\section{2-(4-hydroxyphenyl)-3-(4-(3-(6-methoxybenzo[d]thiazol-2-yl)-2-methyl-4,7-dioxo-2,3,4,7-} tetrahydro-1,3-oxazepin-2-yl)phenyl)-2-methyl-2,3-dihydro-1,3-oxazepine-4,7-dione

FT-IR spectrum data for the compound (4) show peak at $3286 \mathrm{~cm}^{-1}$ for $(\mathrm{OH}), 3070 \mathrm{~cm}^{-}$ 1 for $(\mathrm{Ar}-\mathrm{H}), 2962 \mathrm{~cm}^{-1}$ for $(\mathrm{C}-\mathrm{H})$ in $\mathrm{CH} 3,1636 \mathrm{~cm}^{-1}$ for $(\mathrm{C}=0), 1604 \mathrm{~cm}^{-1}$ for $(\mathrm{C}=\mathrm{N}), 1506 \mathrm{~cm}^{-1}$ for $(\mathrm{C}=\mathrm{C}), 1226 \mathrm{~cm}^{-1}$ for (C-S). ${ }^{1} \mathrm{HMNR}$ spectrum data of compound (4) show 2.50ppm (DMSO) 10.3(1H) $(\mathrm{OH}), 3.7(2 \mathrm{H})(\mathrm{C}=0)-\mathrm{CH})$,3.8(2H)(O-(C=0)CH), , $2.4 \mathrm{ppm}$ (S ,3H, (OCH3)) , 1.2,0.8ppm (S ,6H, (CH3)), 6.2-7.9 ppm(11H)(Ar-H). The ${ }^{13} \mathrm{C}-\mathrm{NMR}$ spectrum data (DMSO) compound (4) show : $28 \mathrm{ppm}\left(\mathrm{C}_{31}, \mathrm{C}_{32}\right), 85 \mathrm{ppm}\left(\mathrm{C}_{11}, \mathrm{C}_{22}\right), 80 \mathrm{ppm}\left(\mathrm{C}_{10}, \mathrm{C}_{21}\right)$, 58ppm $\left(\mathrm{C}_{30}\right)$, 168ppm ( $\left.\mathrm{C}_{1}\right), 191 \mathrm{ppm}\left(\mathrm{C}_{12}, \mathrm{C}_{23}\right), 180 \mathrm{ppm}\left(\mathrm{C}_{9}, \mathrm{C}_{20}\right), 154 \mathrm{ppm}(\mathrm{C} 30), 110-150\left(\mathrm{C}_{\text {Arom }}\right)$

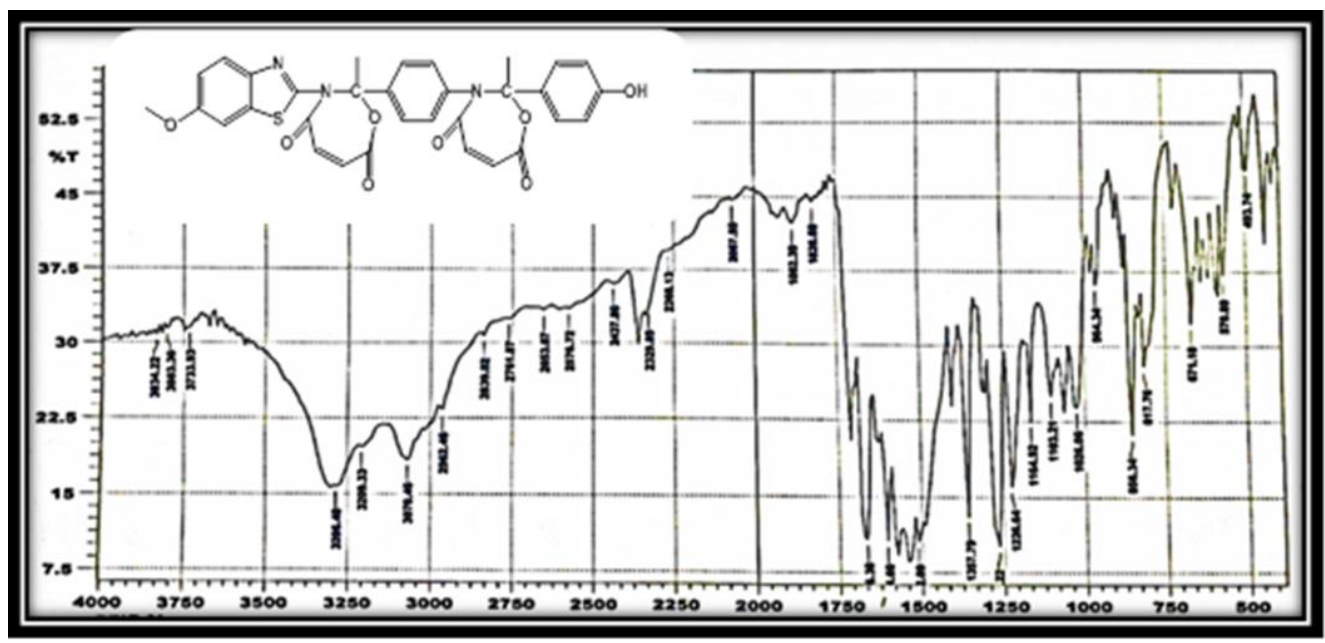

Fig(10) FTIR spectrum of compound(4) 

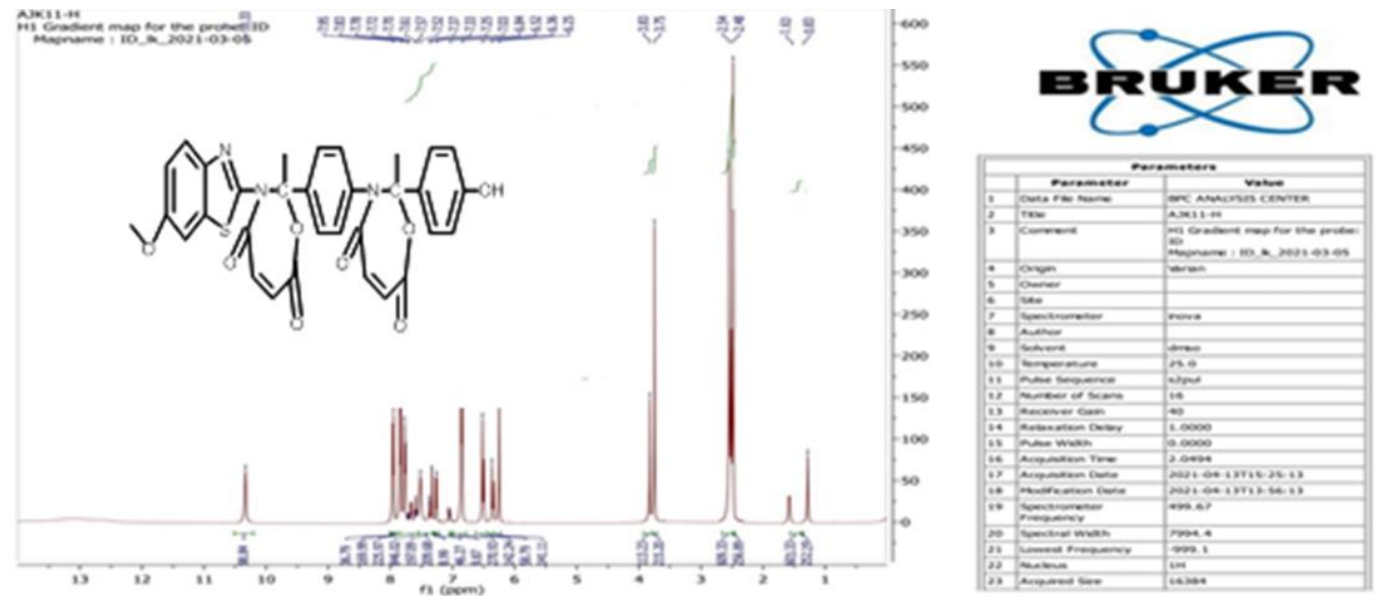

Fig(11)( ${ }^{1}$ H-NMR) spectrum of compound(4)

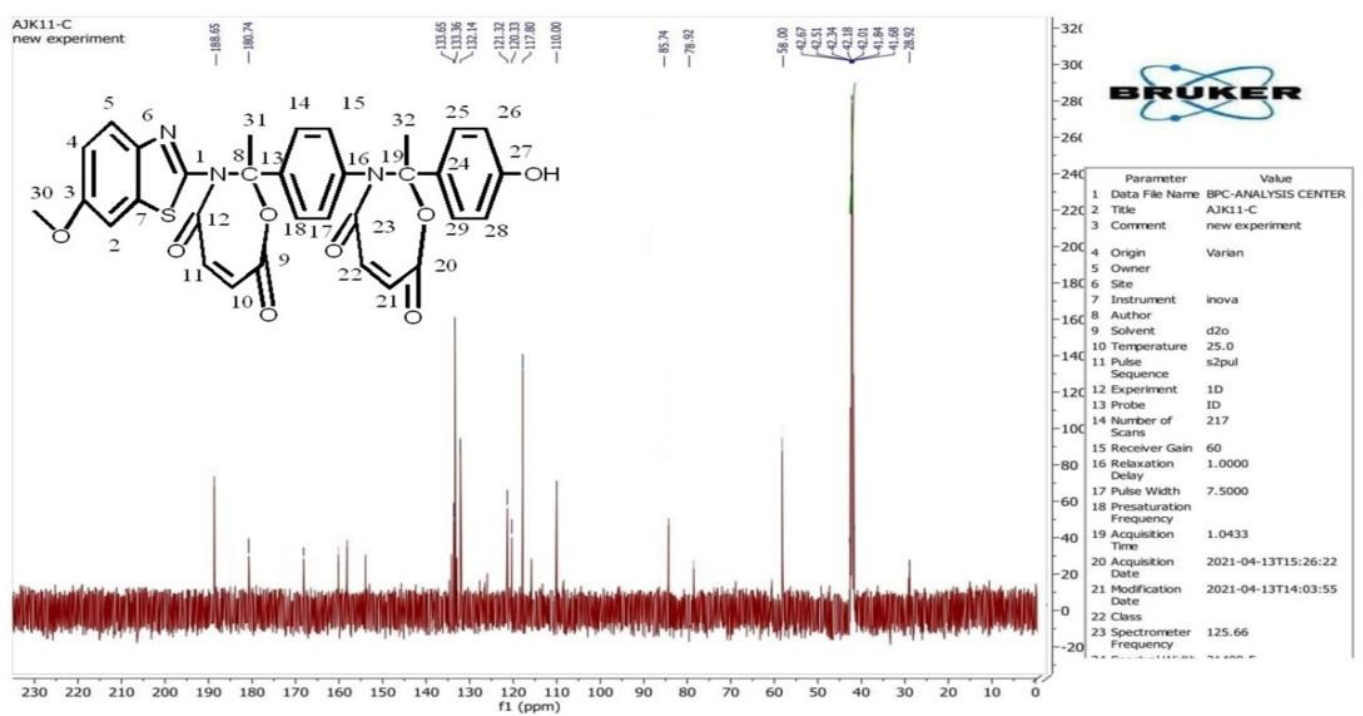

Fig(12) $\left({ }^{13} \mathrm{C}-\mathrm{NMR}\right)$ spectrum of compound(4)

\section{Characterization of compound (5)}

\section{2-(4-hydroxyphenyl)-3-(4-(3-(6-methoxybenzo[d]thiazol-2-yl)-2-methyl-4,7-dioxo-1,3-oxazepan-2-} yl)phenyl)-2-methyl-1,3-oxazepane-4,7-dione

FT-IR spectrum data for the compound (5) show peak at $3322 \mathrm{~cm}^{-1}$ for $(\mathrm{OH})$, $3255 \mathrm{~cm}^{-1}$ for $(\mathrm{Ar}-\mathrm{H}), 2923 \mathrm{~cm}^{-1}$ for $(\mathrm{C}-\mathrm{H})$ in $\mathrm{CH} 3,1656 \mathrm{~cm}^{-1}$ for $(\mathrm{C}=0), 1608 \mathrm{~cm}^{-1}$ for $(\mathrm{C}=\mathrm{N})$, $1434 \mathrm{~cm}^{-1}$ for $(\mathrm{C}=\mathrm{C}), 1226 \mathrm{~cm}^{-1}$ for (C-S). ${ }^{1} \mathrm{HMNR}$ spectrum data of compound (5) show $2.50 \mathrm{ppm}(\mathrm{DMSO}), 2.6 \mathrm{ppm} \quad(\mathrm{S}, 3 \mathrm{H},(\mathrm{OCH} 3)), 2.09 \mathrm{ppm} \quad(\mathrm{S}, 6 \mathrm{H},(\mathrm{CH} 3)), 10.3(1 \mathrm{H})(\mathrm{OH})$, 3.7,3-8 ppm $(4 \mathrm{H})(\mathrm{O}-(\mathrm{C}=\mathrm{H})-\mathrm{CH}), 3.7 .2 .7(4 \mathrm{H})(\mathrm{C}=0) \mathrm{C}-\mathrm{H}, 6.0-7.9 \mathrm{ppm} \quad(11 \mathrm{H}, \mathrm{Ar}-\mathrm{H}))$. The ${ }^{13} \mathrm{CNMR}^{-}$ spectrum data (DMSO) compound (5) show : 11 ppm $\left(\mathrm{C}_{31}, \mathrm{C}_{32}\right), 28.4 \mathrm{ppm}\left(\mathrm{C}_{11}, \mathrm{C}_{22}\right), 28.8 \mathrm{ppm}$ 

$\left(\mathrm{C}_{10}, \mathrm{C}_{21}\right), 38 \mathrm{ppm}\left(\mathrm{C}_{30}\right), 48 \mathrm{ppm} \quad\left(\mathrm{C}_{19}\right), 58 \mathrm{ppm}\left(\mathrm{C}_{8}\right)$ 197ppm( $\left.\mathrm{C}_{9,}, \mathrm{C}_{20}\right), 198 \mathrm{ppm}\left(\mathrm{C}_{23}, \mathrm{C}_{12}\right), 164,108$ $\operatorname{ppm}\left(\mathrm{C}_{\text {Arom }}\right)$

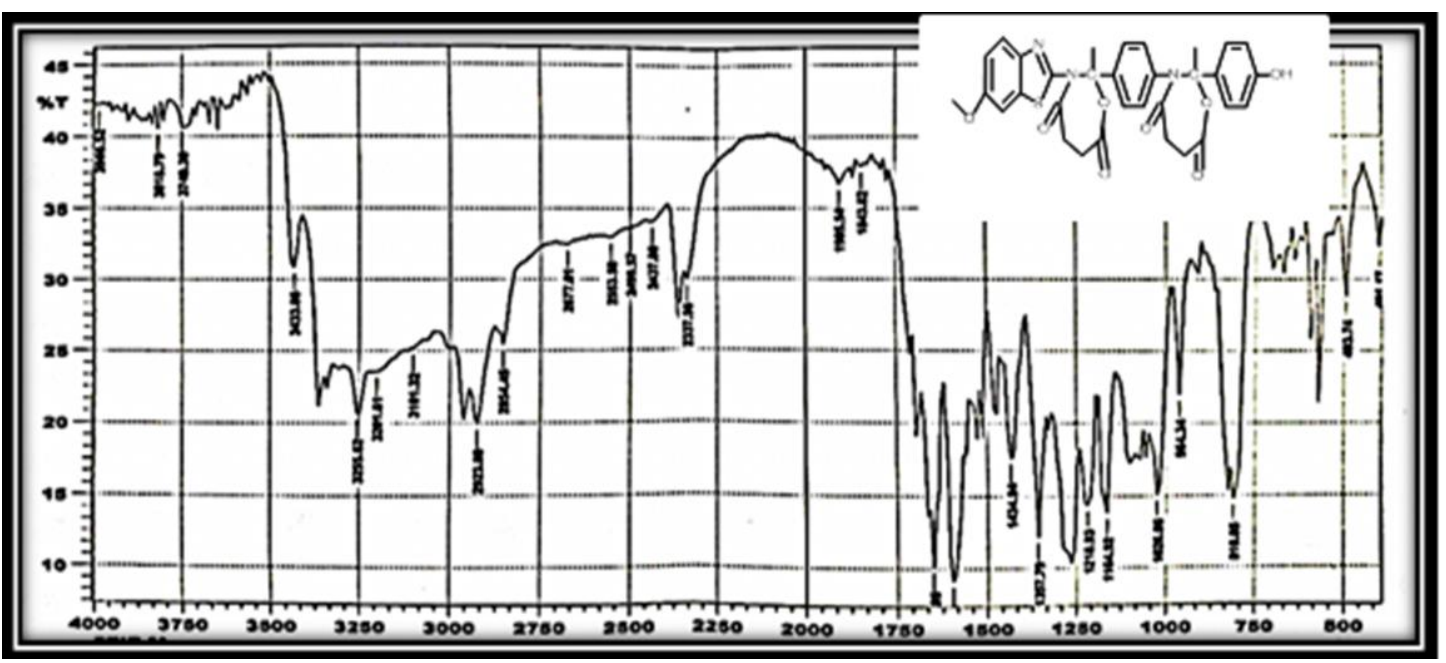

Fig(13) FTIR spectrum of compound(5)
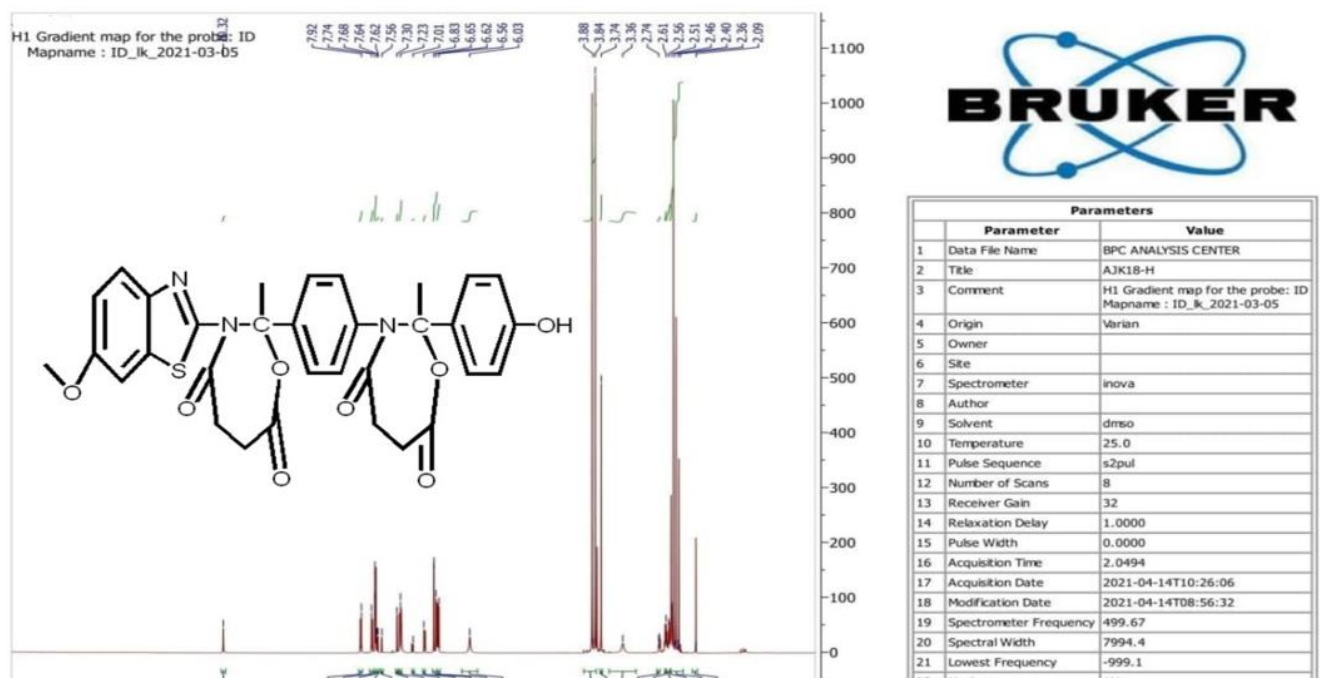

Fig(14)( ${ }^{1}$ H-NMR) spectrum of compound(5) 


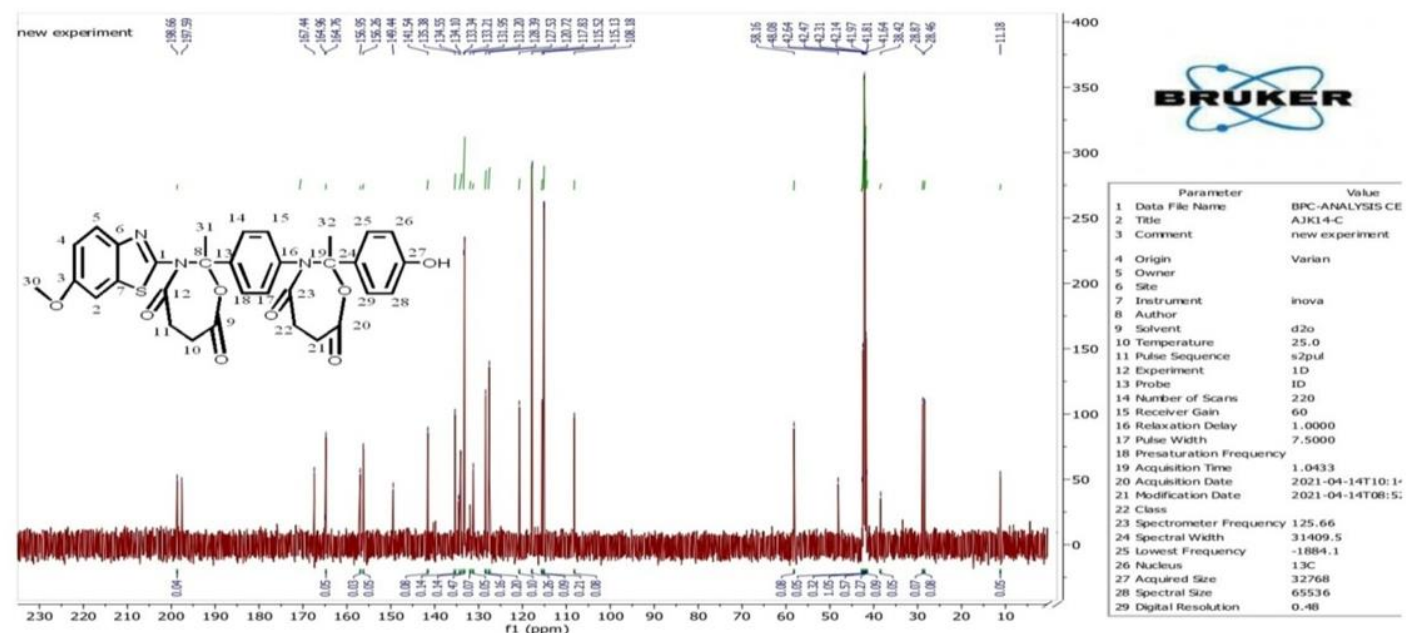

Fig(15) $\left({ }^{13} \mathrm{C}-\mathrm{NMR}\right)$ spectrum of compound(5)

\section{Characterization of compound (6)}

\section{2-(4-hydroxyphenyl)-3-(4-(3-(6-methoxybenzo[d]thiazol-2-yl)-2-methyl-4-oxo-1,2,3,4-}

tetrahydroquinazolin-2-yl)phenyl)-2-methyl-2,3-dihydroquinazolin-4(1H)-one

FT-IR spectrum data for the compound (6) show peak at $3247 \mathrm{~cm}^{-1}$ for (OH) $3109 \mathrm{~cm}^{-1}$ for $(\mathrm{Ar}-\mathrm{H}), 3363 \mathrm{~cm}^{-1}$ for $(-\mathrm{NH}), 2970 \mathrm{~cm}^{-1}$ for $(\mathrm{C}-\mathrm{H})$ in $\mathrm{CH} 3,1650 \mathrm{~cm}^{-1}$ for $(\mathrm{C}=0), 1605 \mathrm{~cm}^{-1}$ for $(\mathrm{C}=\mathrm{N}), 1512 \mathrm{~cm}^{-1}$ for $(\mathrm{C}=\mathrm{C}), 1280 \mathrm{~cm}^{-1}$ for $(\mathrm{C}-\mathrm{S})$. ${ }^{1} \mathrm{HMNR}$ spectrum data of compound (5) show 2.50ppm (DMSO), $3.8 \mathrm{ppm}$ (S ,2H,NH), 3.7ppm (S ,3H, (OCH3)), 1.2,2. 1 ppm (S,6H, CH3), $\quad 6.2-7.9 \mathrm{ppm}(\mathrm{M}, 19 \mathrm{H}, \mathrm{Ar}-\mathrm{H})), 10.6(1 \mathrm{~h})(\mathrm{OH})$. The ${ }^{13}$ CNMR spectrum data (DMSO) compound (6) show : 197 ppm $\left(\mathrm{C}_{15}, \mathrm{C}_{29}\right), 28.4 \mathrm{ppm}\left(\mathrm{C}_{37}, \mathrm{C}_{38}\right)$, , 28.2ppm $\left(\mathrm{C}_{36}\right)$, 58ppm $\left(\mathrm{C}_{9}, \mathrm{C}_{22}\right)$, $.167 \mathrm{ppm}\left(\mathrm{C}_{1}\right),(108-156)\left(\mathrm{C}_{\text {Arom }}\right)$.

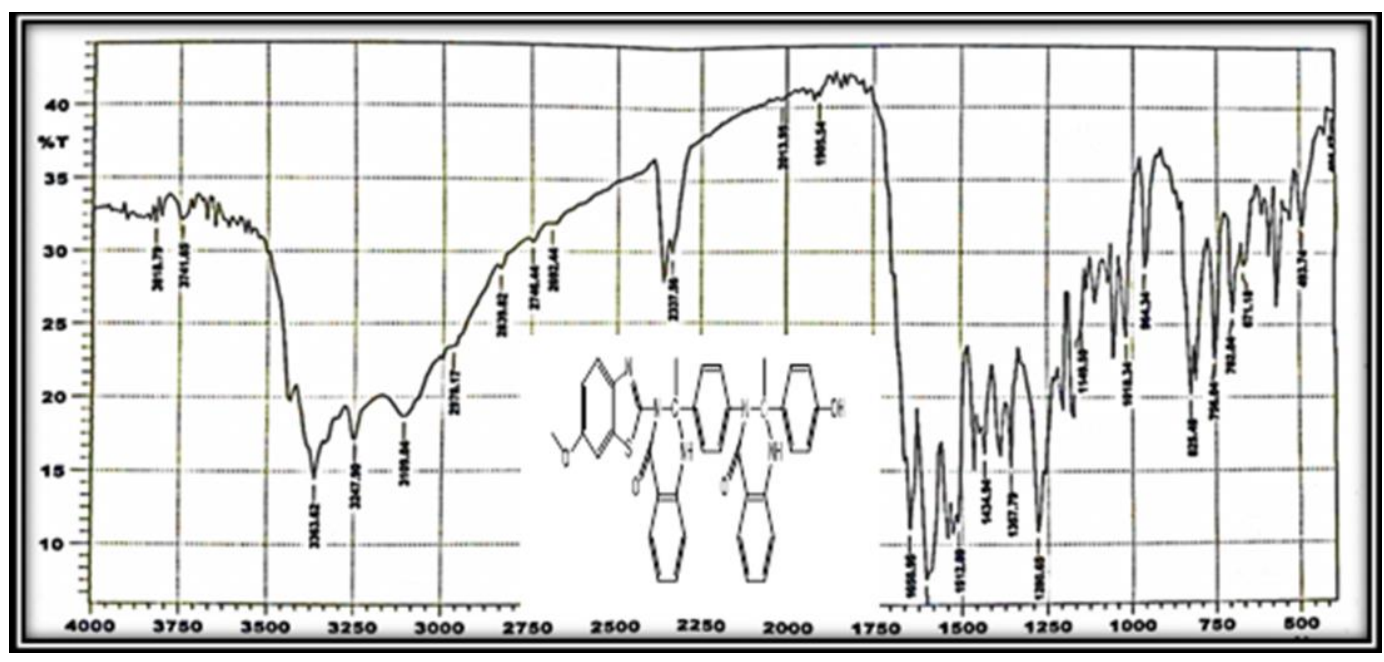

Fig(16) FTIR spectrum of compound(6) 
Fig(17)( ${ }^{1}$ H-NMR) spectrum of compound(6)

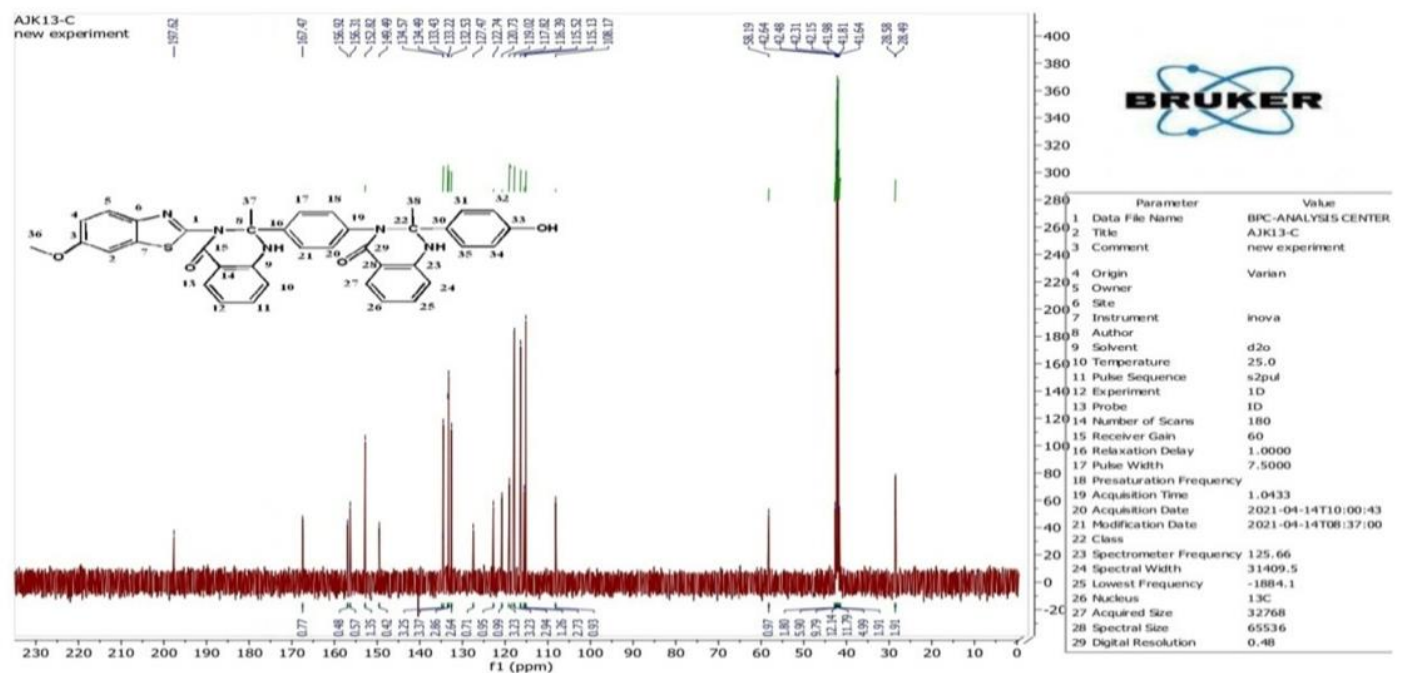

Fig(18) $\left({ }^{15} \mathrm{C}-\mathrm{NMR}\right)$ spectrum of compound(6)

\section{Characterization of compound (7)}

2-(4-hydroxyphenyl)-3-(4-(3-(6-methoxybenzo[d]thiazol-2-yl)-2-methyl-4-oxo-3,4-dihydro-2Hbenzo[e][1,3]thiazin-2-yl)phenyl)-2-methyl-2,3-dihydro-4H-benzo[e][1,3]thiazin-4-one

FT-IR spectrum data for the compound (7) show peak at $3440 \mathrm{~cm}^{-1}$ for $(\mathrm{OH}) 3078 \mathrm{~cm}^{-1}$ for $(\mathrm{Ar}-\mathrm{H}), 2923 \mathrm{~cm}^{-1}$ for $(\mathrm{C}-\mathrm{H})$ in $\mathrm{CH} 3,1650 \mathrm{~cm}^{-1}$ for $(\mathrm{C}=0), 1564 \mathrm{~cm}^{-1}$ for $(\mathrm{C}=\mathrm{C}), 1253 \mathrm{~cm}^{-}$ 1 for (C-S). ${ }^{1}$ HMNR spectrum data of compound (7) show 2.52ppm (DMSO), 3.8ppm (S ,3H, (OCH3) , 0.8- 1.2ppm (S ,6H, (CH3)), 6.0-7.9 ppm (M ,19H, (Ar-H)), 10.3 ppm (1H)(OH) .The ${ }^{13}$ CNMR spectrum data ( DMSO) compound (7) show : 198 ppm $\left(\mathrm{C}_{15}, \mathrm{C}_{29}\right)$ ,28.4,28.9ppm $\left(\mathrm{C}_{37}, \mathrm{C}_{38}\right)$, 31ppm $\left(\mathrm{C}_{36}\right)$, 58pm $\left(\mathrm{C}_{8}, \mathrm{C}_{22}\right), 167 \mathrm{ppm}\left(\mathrm{C}_{1}\right)$, , 108-164 ppm $9\left(\mathrm{C}_{\text {Arom }}\right)$

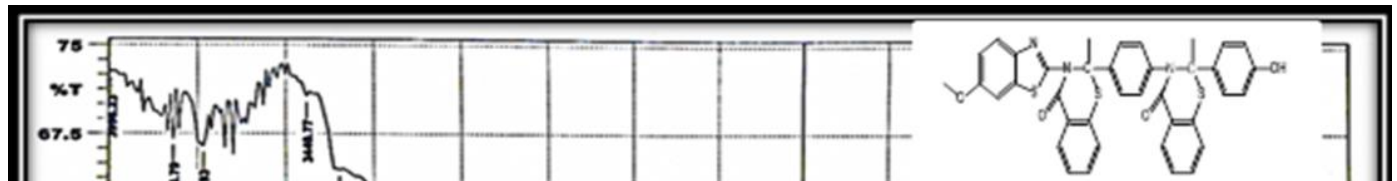


Fig(19) FTIR spectrum of compound(7)
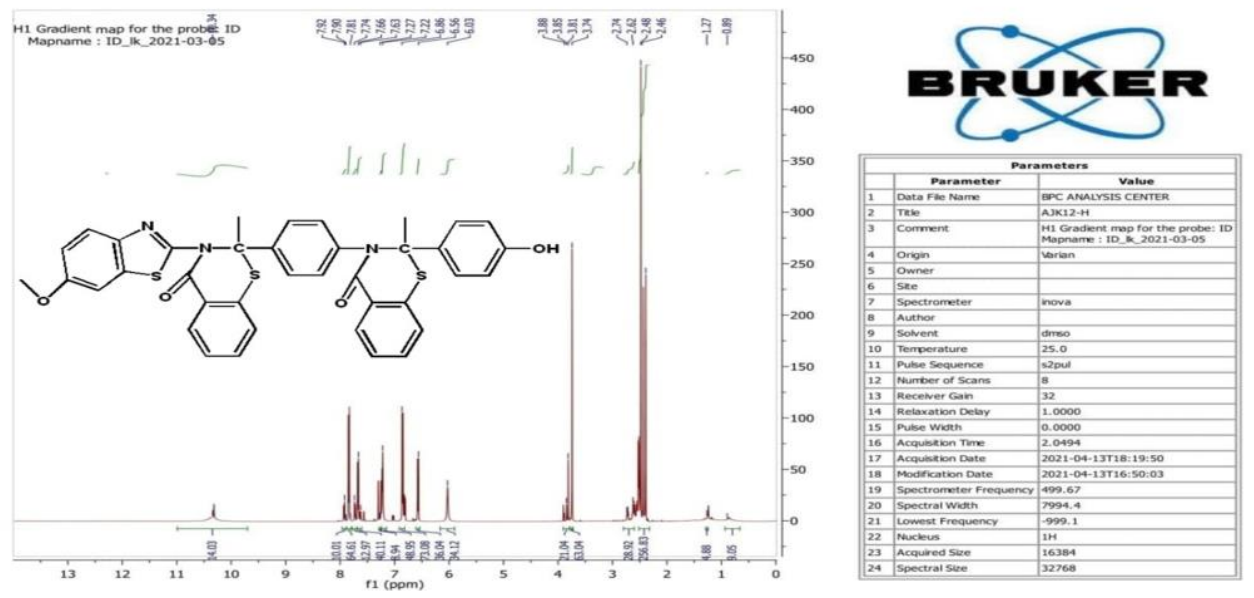

Fig(20)( ${ }^{1}$ H-NMR) spectrum of compound(7)
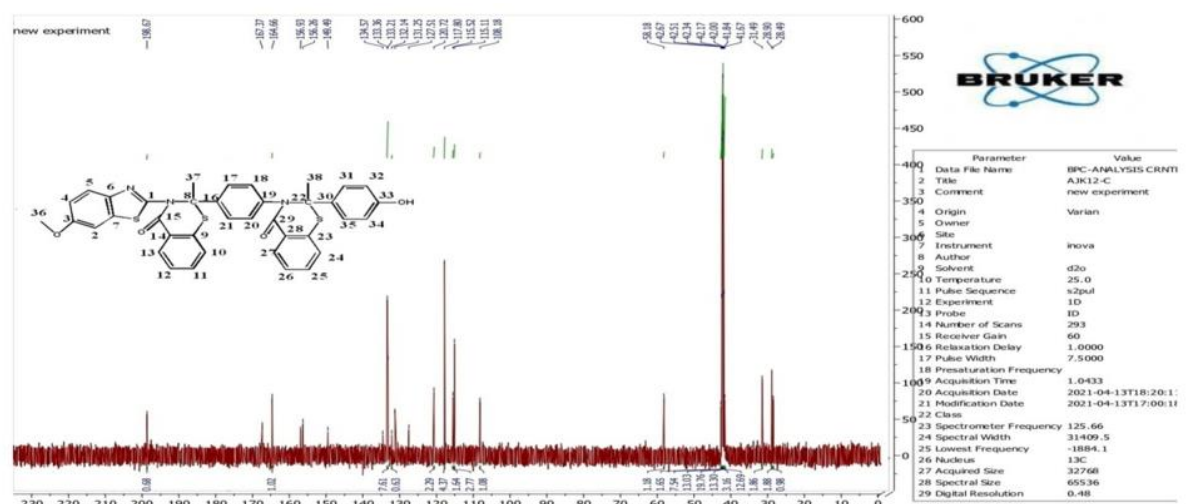

Fig(21) $\left({ }^{13} \mathrm{C}-\mathrm{NMR}\right)$ spectrum of compound(7)

\section{Biological activity}

\section{1 antibacterial}


The results show that derivetives reduce significant antibacterial effectiveness. against bacteria "staphylococcus aurous And Escherichia coli" . the compounds. That show good activity are $(1,2,3,4,5,6,7)$ against (staphylococcus aurous), and compound that show very good activity are (2) against (staphylococcus aurous) , the results of the antibacterial activity are shown in the fig (22)

\begin{tabular}{|c|c|c|c|c|}
\hline Comp.NO & Staph aureus & Mm & E.Coli & Mm \\
\hline 1 & ++ & 15 & +++ & 22 \\
\hline 2 & +++ & 21 & ++ & 22 \\
\hline 3 & ++ & 14 & ++ & 11 \\
\hline 4 & ++ & 14 & ++ & 19 \\
\hline 5 & + & 7 & ++ & 16 \\
\hline 6 & ++ & 15 & + & 14 \\
\hline 7 & +++ & 20 & ++ & 13 \\
\hline
\end{tabular}

Table (1) the results of the antibacterial activity for (1-7) derivatives good active"

$"+=(5-10) \mathrm{mm}=$ slightly active, $\quad++=(11-20) \mathrm{mm}$ moderately, $\quad+++=$ More than 20,

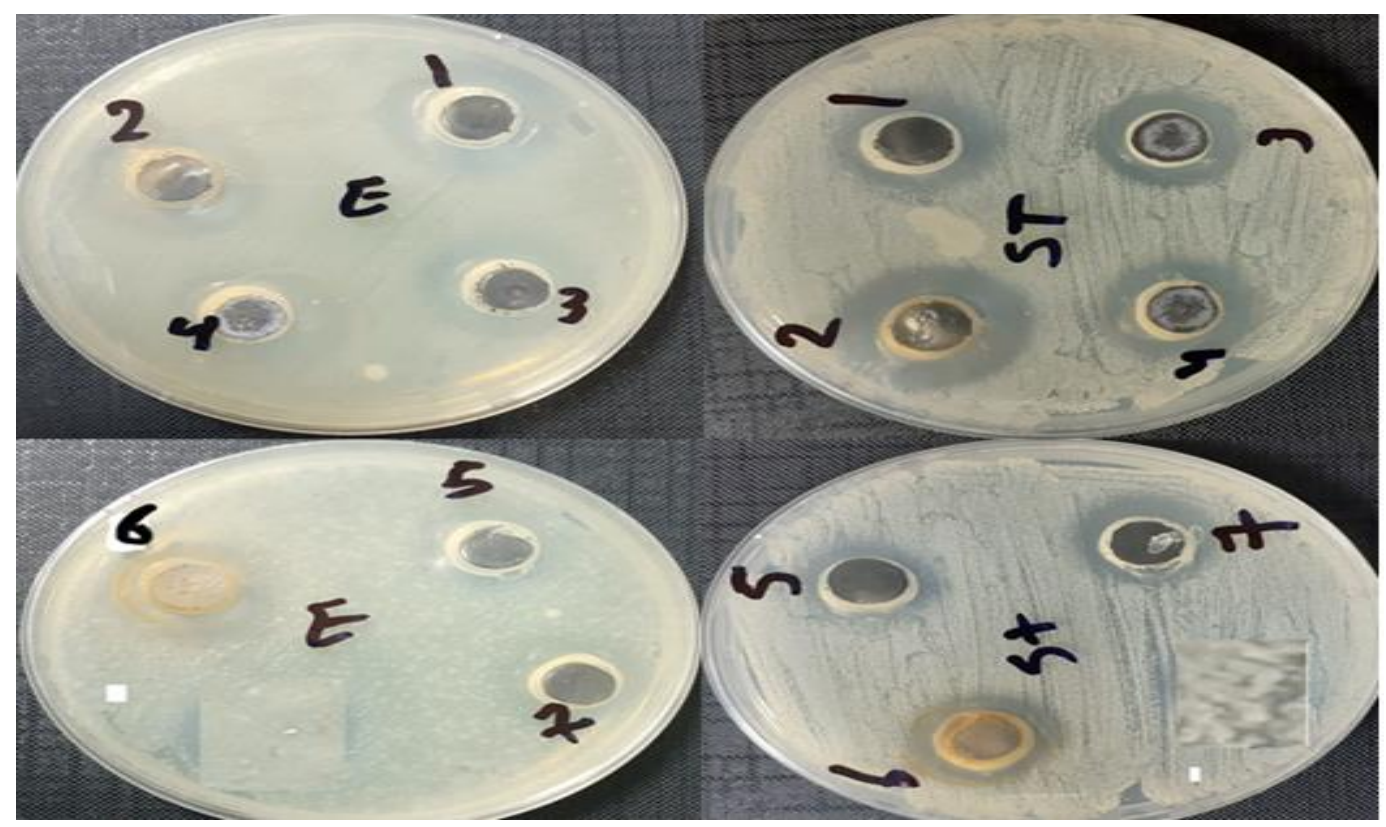

Fig (22) effect compounds (Staph aurous) aginst and (E.Coli) aginst 


\section{References}

1- Alam S.A.M. F., Ahmad T., Nazmuzzaman M., Rahman S., Ray S. K., Sharifuzzaman M., Karim M. R., Alam M. G., Ajam M. M., Maitra P., Mandol D., Uddin M. E., Ahammed T., International Journal of Research Studies in Biosciences (IJRSB) ,5(7), (2017), PP 18-24.

2- Chen X-Y, Li Z-H, Liu J-Q, Wang X-S. Copper-Catalyzed Synthesis of Dibenzo [b, f] imidazo [1, 2d][1, 4] oxazepine Derivatives via a Double Ullmann Coupling Reaction. Synthesis. 2019;51(07):1662-8.

3- Gómez, Elena, et al. "Physical properties of pure 1-ethyl-3-methylimidazolium ethylsulfate and its binary mixtures with ethanol and water at several temperatures." Journal of Chemical \& Engineering Data 51.6 (2006): 2096-2102.

4- Iman K. Naeem, Ezzat H. Zimam, Der Pharma Chemica, 9(21), (2017), pp. 86-93 .

5- Julekha A. Shaikh, International Letters of Chemistry, Physics and Astronomy, 17(3), (2014), pp. 272-280

6- Girly Tony, Meena Chandran, A. R Bhat, K. Krishnakumar, Journal of Pharmacy Research, 8(2), (2014), pp. 136-138.

7- Khan, Muhammad I., Saima Gul, and Murad Ali Khan. "Schiff Bases and Their Metallic Derivatives: Highly Versatile Molecules with Biological and Abiological Perspective." Stability and Applications of Coordination Compounds. IntechOpen 2019

8- Kuddushi, Mohammad Muzammil Y., et al. "Synthesis and characterization of Schiff base aniline with 5-bromo-2-hydroxyl benzaldehyde and their metal complexes." International Journal of Recent Scientific Research 9 (2018): 26026-26030.

9- Mumtaz, Amina, et al. "Synthesis and characterization of new Schiff base transition metal complexes derived from drug together with biological potential study." J Nucl Med Radiat Ther 7.310 (2016): 2 .

10- Mohammed, Mieaad, et al. "New Azomethine-Azo heterocyclic ligands via cyclization of ester." Research Journal of Pharmacy and Technology 11.6 (2018): 2555-2560. 
11- Reyes-Arellano, Alicia, Omar Gómez-García, and Jenifer Torres-Jaramillo. "Synthesis of azolines and imidazoles and their use in drug design." Med Chem (Los Angeles) 6 (2016): 561-570.

12- Shaabani S, Shaabani A, Kucerakova M, Dusek M. A One-Pot synthesis of oxazepinequinazolinone bis-heterocyclic scaffolds via isocyanide-based three-component reactions. Frontiers in Chemistry. 2019;7:623.

13- Slassi, Siham, et al. "Imidazole and azo-based Schiff bases ligands as highly active antifungal and antioxidant components." Heteroatom Chemistry 2019 (2019). $\quad$ 10- Zora M, Dikmen E, Kelgokmen Y. One-pot synthesis of iodine-substituted 1, 4-oxazepines. Tetrahedron Letters. 2018;59(9):823-7.

14- Vimal Patel1, Pranav Trivedi, Hardik Gohel, Disha Khetani, IJAPBC, 3(4), (2014), pp. 999-1003.

15-Zaki N. Kadhim, J. Mater . Environ. Sci., 6(3), (2015), pp. 693-698.

16- Zena. G. Alrecabi ,Redhab A. J. Alfraiji, Suaad M.H. Al-Majidi, Iraqi Journal of Science, 58(3c), (2017), pp. 1565-1579.

17- Zora M, Dikmen E, Kelgokmen Y. One-pot synthesis of iodine-substituted 1, 4-oxazepines. Tetrahedron Letters. 2018;59(9):823-7. 